\title{
Equilibrium beam distribution and quantum lifetime in the presence of a single nonlinear resonance
}

\author{
Alexander W. Chao \\ Stanford Linear Accelerator Center, Stanford University, Stanford, California 94309, USA
}

(Received 27 June 2003; published 16 September 2003)

\begin{abstract}
In the proximity of a nonlinear resonance $\nu \approx \frac{m}{n}$, the beam distribution in a storage ring is distorted depending on how close is the resonance and how strong is the resonance strength. In the 1-dimensional case, it is well known that the particle motion near the resonance can be described in a smooth approximation by a Hamiltonian of the form $\left(\nu-\frac{m}{n}\right) J+D_{\nu}(J)+f_{1}(\phi, J)$, where $(\phi, J)$ are the phase space angle and action variables, $D_{\nu}$ is the detuning function, and $f_{1}$ is an oscillating resonance term. In a proton storage ring, the equilibrium beam distribution is readily solved to be any function exclusively of the Hamiltonian. For an electron beam, this is not true and the equilibrium distribution is more complicated. This paper solves the Fokker-Planck equation near a single resonance for an electron beam in a storage ring. The result is then applied to obtain the quantum lifetime of an electron beam in the presence of this resonance. Resonances due to multipole fields and due to the beam-beam force are considered as examples.
\end{abstract}

DOI: 10.1103/PhysRevSTAB.6.094001

PACS numbers: 29.27.-a, 41.85.-p

\section{INTRODUCTION AND SUMMARY}

Particle motion in a storage ring is seriously perturbed when the particle's tune $\nu$ (defined as the particle's natural oscillation frequency divided by its revolution frequency around the ring) is close to a fractional value, i.e., when a nonlinear resonance condition $\nu \approx \frac{m}{n}$ is fulfilled. In particular, the beam distribution in phase space can be significantly distorted depending on how close is the resonance and how strong is the resonance strength.

In the 1-dimensional case, it is well known that the particle motion can be described in a smooth approximation [1] by a Hamiltonian of the form

$$
K=\frac{1}{R}\left[\left(\nu-\frac{m}{n}\right) J+D_{\nu}(J)+f_{1}(\phi, J)\right],
$$

where $(\phi, J)$ are the phase space angle and action variables, $2 \pi R$ is the storage ring circumference, $D_{\nu}$ is the detuning function, and $f_{1}(\phi, J)$ is some resonance structure function which is purely oscillatory in the sense that it is periodic in $\phi$ with period $2 \pi / n$ and its average over $\phi$ vanishes.

In a proton storage ring, the equilibrium beam distribution near a resonance is readily obtained once the smooth Hamiltonian is available; it is given by any function exclusively of $K$, i.e.,

$$
\psi(\phi, J)=\text { any function } \psi_{0}(K),
$$

where the function $\psi_{0}$ is determined by the initial condition of the beam. Any function of $K$ is a possible equilibrium distribution for a proton beam.

For an electron beam, however, this is no longer the case and the equilibrium distribution is more complicated. In addition to the Hamiltonian dynamics, we also include the slow radiation damping and quantum diffu- sion effects caused by synchrotron radiation [2]. In the absence of resonances, the equilibrium distribution is also well known and is given by the Gaussian distribution

$$
\psi(\phi, J)=e^{-J / J_{0}},
$$

where $J_{0}$ is the nominal beam emittance determined by a balance between the damping and diffusion effects. (The distribution is exponential in $J$, but is Gaussian in $x$ and $p$. Its normalization is $\int_{0}^{\infty} d J \int_{0}^{2 \pi} d \phi \psi=2 \pi J_{0}$.) The question therefore remains as to what will the electron beam distribution become when there is a nonlinear resonance nearby. Simple physical arguments can be constructed to show that a simple Gaussian of the new Hamiltonian such as $\psi \sim e^{-K}$ will not do the job. (For example, as $D_{\nu}$ is bound to dominate over the other terms in the Hamiltonian at large $J$, the distribution becomes divergent at large $J$ and is unnormalizable if $D_{\nu}<0$. Also, as another example, consider the case away from resonances, for which one has $f_{1}=0$. In that case, one would expect the beam distribution to be given by (3) regardless of $D_{\nu}$. This expected behavior is not observed by $\psi \sim e^{-K}$.)

In this paper, we will first establish the Fokker-Planck equation near a single resonance for an electron beam in a storage ring. By solving this equation to first order in the resonance strength, we find that the equilibrium distribution is given by

$$
\psi(\phi, J)=\exp \left[-\frac{1}{J_{0}}\left(J+\frac{f_{1}(\phi, J)}{\nu-\frac{m}{n}+D_{\nu}^{\prime}(J)}\right)\right] .
$$

The three terms in the new Hamiltonian (1) take on different roles and appear in different places in the equilibrium beam distribution, reflecting their respective physical meanings. 
The result (4) is then applied to obtain the quantum lifetime of an electron beam in the presence of a nearby single resonance. Quantum lifetime is the lifetime of the electron beam when particles are brought by the diffusion effect to a maximum allowed amplitude (representing an absorbing wall in the physical $x$ space) and are lost from the beam. We find that due to the proximity to the nonlinear resonance, the quantum lifetime is shortened by a factor

$$
\exp \left(\frac{\hat{f}_{1}(\hat{J})}{J_{0}\left|\nu-\frac{m}{n}+D_{\nu}^{\prime}(\hat{J})\right|}\right)
$$

compared with the nominal expression [2]. In Eq. (5), $\hat{J}=$ $A^{2} / 2 \beta$ with $A$ the maximum allowed value of $x, \beta$ the $\beta$ function at the aperture limit, and $\hat{f}_{1}(J)$ the oscillation amplitude of $f_{1}(\phi, J)$.

In Sec. II, we give a brief summary of the well-known smooth approximation in the 1-dimensional case. Section III derives the Fokker-Planck equation in the smooth approximation. Section IV solves the FokkerPlanck equation for the equilibrium beam distribution with weak resonances and slow damping and diffusion. Section V derives an expression for the quantum lifetime. Section VI applies these results to specific examples, first to the case of a nonlinear magnet field error, and then to the case of a 1-dimensional beam-beam perturbation.

\section{SMOOTH APPROXIMATION NEAR A SINGLE RESONANCE}

Consider 1-dimensional dynamics of a particle in a storage ring. Let the particle motion be described by the Hamiltonian in the $(x, p)$ phase space

$$
H(x, p)=\frac{p^{2}}{2}+\frac{K(s) x^{2}}{2}+\frac{f(x, s)}{R}
$$

where $K(s)$ specifies the linear focusing system of the storage ring, and $f(x, s)$ represents a weak nonlinear perturbation. Both $K(s)$ and $f(x, s)$ are periodic in $s$ with period $2 \pi R$.

In the presence of one and only one single resonance $\nu=\frac{m}{n}$, we follow Ref. [3] and make a canonical transformation similar to the Courant-Snyder transformation from $(x, p)$ to $(\phi, J)$. We use the generating function

$$
\begin{aligned}
G(x, \phi) & =-\frac{x^{2}}{2 \beta(s)}\left[\tan \phi_{1}-\frac{\beta^{\prime}(s)}{2}\right], \\
\phi_{1} & =\phi+\frac{m}{n} \frac{s}{R}+\int_{0}^{s} d s^{\prime}\left(\frac{1}{\beta\left(s^{\prime}\right)}-\frac{\nu}{R}\right),
\end{aligned}
$$

where $\beta(s)$ is the periodic Courant-Snyder $\beta$ function and $\beta^{\prime}(s)=(d \beta / d s)$. With this generating function, we obtain

$$
\begin{aligned}
& x=\sqrt{2 J \beta(s)} \cos \phi_{1}, \\
& p=-\sqrt{\frac{2 J}{\beta(s)}}\left[\sin \phi_{1}-\frac{\beta^{\prime}(s)}{2} \cos \phi_{1}\right],
\end{aligned}
$$

or

$$
J=\frac{x^{2}}{2 \beta}+\frac{\beta}{2}\left(p-\frac{\beta^{\prime}}{2 \beta} x\right)^{2}, \quad \tan \phi_{1}=-\beta \frac{p}{x}+\frac{\beta^{\prime}}{2} .
$$

The new Hamiltonian is

$$
\begin{aligned}
K(\phi, J) & =H+\frac{\partial G}{\partial s} \\
& =\frac{J}{R}\left(\nu-\frac{m}{n}\right)+\frac{1}{R} f\left[\sqrt{2 J \beta(s)} \cos \phi_{1}, s\right],
\end{aligned}
$$

where use has been made of $2 \beta \beta^{\prime \prime}-\beta^{\prime 2}+4 \beta^{2} K=4$ [3].

In the smooth approximation, we drop fast oscillating terms from Eq. (10). This means the Hamiltonian is approximated by replacing the $f$ term in Eq. (10) by its average over $s$ over the range $(0,2 \pi n R)$. The new Hamiltonian therefore becomes

$$
K(\phi, J) \approx \frac{J}{R}\left(\nu-\frac{m}{n}\right)+\frac{1}{R}\langle f\rangle_{s},
$$

where

$$
\langle f\rangle_{s}=\int_{0}^{2 \pi n R} \frac{d s}{2 \pi n R} f\left[\sqrt{2 J \beta(s)} \cos \phi_{1}, s\right]
$$

is a function of $\phi$ and $J$ but is no longer a function of $s$. In Eq. (12), the integration over $s$ is done by considering $\phi_{1}$ as a function of $s$ while holding $\phi$ fixed. The quantity $\langle f\rangle_{s}$ is a periodic function of $\phi$ with period $2 \pi / n$.

The smooth approximation therefore allows the replacement of a time-dependent Hamiltonian (6) by a time-independent Hamiltonian (11). The particle motion is described by applying the Hamilton's equations to the new Hamiltonian. Since this new Hamiltonian is independent of $s$, it is a constant of the motion. One may note that the Courant-Snyder transformation for a linear system [when $f(x, s)=0$ ] is in fact a special case of smooth approximation in the sense that the $s$-dependent focusing function $K(s)$ has been replaced by the tune $\nu$. The linear case, on the other hand, enjoys the distinction that the Courant-Snyder transformation is exact.

The function $\langle f\rangle_{s}$ is periodic in $\phi$ with period $2 \pi / n$. As $\phi$ varies, $\langle f\rangle_{s}$ may be decomposed into a constant part given by

$$
D_{\nu}(J)=\frac{1}{4 \pi^{2}} \int_{0}^{2 \pi} d \xi \int_{0}^{2 \pi} \frac{d s}{R} f\left\{\sqrt{2 J \beta(s)} \cos \left[\xi+\int_{0}^{s} d s^{\prime}\left(\frac{1}{\beta\left(s^{\prime}\right)}-\frac{\nu}{R}\right)\right], s\right\}
$$

plus an oscillating part $f_{1}(\phi, J)$, i.e., 


$$
\langle f\rangle_{s}=D_{\nu}(J)+f_{1}(\phi, J)
$$

which in turn gives Eq. (1). As we shall see, the two terms $D_{\nu}$ and $f_{1}$ play quite different roles in the beam dynamics.

\section{FOKKER-PLANCK EQUATION}

The Fokker-Planck equation in the $(x, p)$ space is given by [4]

$$
\frac{\partial \psi}{\partial s}+\frac{\partial H}{\partial p} \frac{\partial \psi}{\partial x}-\left(\frac{\partial H}{\partial x}+2 \alpha p\right) \frac{\partial \psi}{\partial p}-\frac{D}{\beta(s)} \frac{\partial^{2} \psi}{\partial p^{2}}-2 \alpha \psi=\frac{\partial \psi}{\partial s}+p \frac{\partial \psi}{\partial x}-\left(K x+\frac{1}{R} \frac{\partial f}{\partial x}+2 \alpha p\right) \frac{\partial \psi}{\partial p}-\frac{D}{\beta(s)} \frac{\partial^{2} \psi}{\partial p^{2}}-2 \alpha \psi=0,
$$

where $\alpha$ and $D$ are the damping and diffusion coefficients. Note that a factor $\beta(s)$ is introduced in the diffusion constant. Strictly speaking, this form of the Fokker-Planck equation assumes that quantum noise occurs in $p$ and not in $x$. However, when the diffusion process is slow, our result does not change if the noise also occurs in $x$. What matters is only the effect of diffusion after averaging out the fast oscillating terms.

We then make the transformation to the $(\phi, J)$ space. The Fokker-Planck equation in the $(\phi, J)$ space becomes, after some algebra,

$$
\begin{gathered}
\frac{\partial \psi}{\partial s}+\frac{1}{R}\left(\nu-\frac{m}{n}\right) \frac{\partial \psi}{\partial \phi}+\left(\frac{1 \partial f}{R \partial x}+2 \alpha p\right)\left(\sqrt{2 J \beta} \sin \phi_{1} \frac{\partial \psi}{\partial J}+\sqrt{\frac{\beta}{2 J}} \cos \phi_{1} \frac{\partial \psi}{\partial \phi}\right)-D\left(2 J \sin ^{2} \phi_{1} \frac{\partial^{2} \psi}{\partial J^{2}}+\frac{\partial \psi}{\partial J}+\sin 2 \phi_{1} \frac{\partial^{2} \psi}{\partial \phi \partial J}-\frac{1}{2 J} \sin 2 \phi_{1} \frac{\partial \psi}{\partial \phi}\right. \\
\left.+\frac{1}{2 J} \cos ^{2} \phi_{1} \frac{\partial^{2} \psi}{\partial \phi^{2}}\right)-2 \alpha \psi=0
\end{gathered}
$$

Damping and diffusion effects are considered to be very slow. If we consider only this very slow evolution of $\psi$, then we can average the Fokker-Planck equation over $s$ (or $\phi_{1}$ ), i.e., average over the fast oscillation. This yields the FokkerPlanck equation under smooth approximation,

$$
\frac{\partial \psi}{\partial s}+\frac{\partial K}{\partial J} \frac{\partial \psi}{\partial \phi}-\frac{\partial K}{\partial \phi} \frac{\partial \psi}{\partial J}-2 \alpha\left(J \frac{\partial \psi}{\partial J}-\frac{\beta^{\prime}}{4} \frac{\partial \psi}{\partial \phi}\right)-D\left(J \frac{\partial^{2} \psi}{\partial J^{2}}+\frac{\partial \psi}{\partial J}+\frac{1}{4 J} \frac{\partial^{2} \psi}{\partial \phi^{2}}\right)-2 \alpha \psi=0
$$

When $f=0$, the equilibrium beam distribution depends only on $J$, and indeed, by solving Eq. (17), we recover the distribution (3) with

$$
J_{0}=\frac{D}{2 \alpha} .
$$

In terms of the unperturbed rms beam size $\sigma_{x}$, we have $J_{0}=\sigma_{x}^{2} / \beta$.

\section{EQUILIBRIUM DISTRIBUTION}

We need to solve Eq. (17) for the equilibrium distribution when $f \neq 0$ in the smooth approximation. To do so, we make two assumptions. We first assume that the resonance is weak in such a way that $f_{1}$ is small. More specifically, as can be seen in the manipulations with the Fokker-Planck equation, we require

$$
\nu-\frac{m}{n}+D_{\nu}^{\prime}(J) \gg \frac{1}{J} f_{1}(\phi, J) .
$$

The left-hand side is the distance of the amplitude-dependent tune $\nu+D_{\nu}^{\prime}(J)$ from the resonance. The righthand side can be considered a resonance width in tune units. This condition (19) applies when either $\nu-\frac{m}{n}$ or the detuning $D_{\nu}^{\prime}$ is larger than the resonance width, but it does not require both to be larger. For example, the analysis will be applicable exactly on resonance $\nu=\frac{m}{n}$, provided that $D_{\nu}^{\prime}$ is sufficiently large. On the other hand, the analysis breaks down when $\nu-\frac{m}{n}+D_{\nu}^{\prime}(J)=0$, i.e., when the amplitude-dependent tune crosses the resonance at some amplitude within the beam distribution.

We also assume that the damping and diffusion effects are very slow, so that

$$
\nu-\frac{m}{n}+D_{\nu}^{\prime}(J) \gg \alpha R=\frac{T_{0}}{2 \pi \tau},
$$

where $T_{0}=2 \pi R / c=$ revolution period, and $\tau=$ $1 / \alpha c=$ radiation damping time.

What assumptions (19) and (20) allow us to do is that, in the Fokker-Planck equation, we may keep only terms that are up to first order in $f_{1}, \alpha$, or $D$. Higher order terms in these parameters, such as terms proportional to $\alpha f_{1}$, can be dropped. [Since $f_{1}, \alpha$ and $D$ are small parameters, this seems to be a reasonable procedure. Nevertheless, a more detailed justification of this procedure can be established by making another canonical transformation from the $(\phi, J)$ coordinate system to a new system $(\Phi, K)$, where the new momentum is just the Hamiltonian itself. The same exercise also provided the hint of the ansatz (21) below.]

With assumptions (19) and (20), it turns out that we may try the ansatz for the equilibrium beam distribution in the form 


$$
\psi=e^{-A(J)-B(J) f_{1}(\phi, J)} .
$$

Substituting Eq. (21) into (17), keeping terms first order in $f_{1}, \alpha$, and $D$, yields the solution

$$
A(J)=\frac{J}{J_{0}}, \quad B(J)=\frac{1}{J_{0}\left[\nu-\frac{m}{n}+D_{\nu}^{\prime}(J)\right]},
$$

where $J_{0}$ is given by Eq. (18). It is remarkable that this equilibrium distribution holds for arbitrary $\nu-\frac{m}{n}, D_{\nu}(J)$, and $f_{1}(\phi, J)$, and for all $J$. We have therefore arrived at Eq. (4). The term involving $f_{1}$ gives rise to island structure in the beam distribution. Near the resonance $\nu \approx \frac{m}{n}$, there will be $n$ islands in the phase space [5].

The normalization is such that, to first order in $f_{1}$,

$$
\int_{0}^{\infty} d J \int_{0}^{2 \pi} d \phi \psi(\phi, J)=2 \pi J_{0} .
$$

The distribution is not normalizable if $f_{1}$ overcomes the detuning, i.e., if the opposite of condition (19) holds.

\section{QUANTUM LIFETIME}

When the storage ring imposes a maximum permitted aperture to the electron beam, the beam will suffer a continuous loss of particles due to quantum diffusion [2]. If the aperture is far from the beam core, this loss will be slow, and the corresponding quantum beam life- time will be long. The beam distribution will still be accurately given by Eq. (4) except immediately near the aperture boundary [6]. In particular, the distribution will be a function of

$$
J_{1}=J+\frac{f_{1}(\phi, J)}{\nu-\frac{m}{n}+D_{\nu}^{\prime}(J)}
$$

which can be considered a distorted action variable and where the second term is purely oscillatory in $\phi$ with period $2 \pi / n$. We shall write the beam distribution with an aperture limit as

$$
\psi(\phi, J, s)=\psi_{1}\left(J_{1}\right) e^{-\left(s / \tau_{q} c\right)}
$$

where $\tau_{q}$ is the quantum lifetime.

The aperture boundary is imposed on the physical amplitude $x$ of particle motion. Let this limit be $A$, i.e., a particle is lost when its physical amplitude reaches $x \geq$ $A$, or equivalently $J \geq A^{2} / 2 \beta$, with $\beta$ the $\beta$ function at the aperture limit. This means that a particle is lost when its $J_{1}$ reaches $J_{1} \geq \hat{J}_{1}$, where

$$
\hat{J}_{1}=\frac{A^{2}}{2 \beta}-\frac{\hat{f}_{1}\left(\frac{A^{2}}{2 \beta}\right)}{\left|\nu-\frac{m}{n}+D_{\nu}^{\prime}\left(\frac{A^{2}}{2 \beta}\right)\right|}
$$

A particle with $J_{1} \geq \hat{J}_{1}$ will have during its motion a moment when its physical $x$ reaches the aperture $x \geq A$.

The beam distribution still obeys the Fokker-Planck Eq. (17), except now at the aperture boundary, the beam distribution will have to satisfy the additional condition

$$
\psi_{1}\left(J_{1}=\hat{J}_{1}\right)=0 \text {. }
$$

With $\psi_{1}$ depending only on $J_{1}$, and keeping terms linear in $f_{1}, \alpha$, and $D$, the Fokker-Planck equation reduces to

$$
-\frac{1}{c \tau_{q}} \psi_{1}-2 \alpha J_{1} \psi_{1}^{\prime}-D\left(J_{1} \psi_{1}^{\prime \prime}+\psi_{1}^{\prime}\right)-2 \alpha \psi_{1}=0
$$

where the prime on $\psi_{1}$ means taking a derivative with respect to $J_{1}$. Integrating Eq. (29) over $J_{1}$ from 0 to $\hat{J}_{1}$ and applying integration by parts, we obtain

$$
\frac{1}{\tau_{q} c} \int_{0}^{\hat{J}_{1}} \psi_{1} d J_{1}+D \hat{J}_{1} \psi_{1}^{\prime}\left(\hat{J}_{1}\right)=0
$$

When the aperture is far from the beam core, i.e., when $\hat{J}_{1} \gg J_{0}, \psi_{1}$ will be accurately given by the unperturbed distribution (4) [2,6]. Near the aperture boundary, $\psi_{1}$ will have to approach zero, but the derivative $\psi_{1}^{\prime}$ will remain very close to the derivative obtained using Eq. (4). (Physically this is because the diffusion rate outward from the beam through the aperture will be the same whether the aperture limit is there or not.) We therefore substitute $\psi_{1}\left(J_{1}\right)=e^{-J_{1} / J_{0}}$ into both terms in Eq. (30), and obtain

$$
\frac{1}{\tau_{q}}=\frac{2 \hat{J}_{1}}{\tau J_{0}} e^{-\hat{J}_{1} / J_{0}}
$$

where $\tau=1 / \alpha c$ is the radiation damping time. When $f_{1}=0$, Eq. (31) reduces to the nominal expression in the absence of the nonlinear resonance [2],

$$
\frac{1}{\tau_{q 0}}=\frac{A^{2}}{\tau \sigma_{x}^{2}} e^{-A^{2} / 2 \sigma_{x}^{2}}
$$

When $f_{1} \neq 0$, one finds that the quantum lifetime in the presence of the resonance is shortened compared with the nominal expression by a factor (5). Note that the argument of the exponent in Eq. (5) does not have to be $\ll 1$ under the constraints (19) and (20). 


\section{EXAMPLE APPLICATIONS}

We now apply the results obtained so far to specific examples. Consider a perturbation of the form

$$
\begin{aligned}
f(x, s) & =\epsilon g(x) \delta_{P}(s), \\
\delta_{P}(s) & =\text { periodic } \delta \text { function } \sum_{k=-\infty}^{\infty} \delta\left(\frac{s}{R}-2 \pi k\right)
\end{aligned}
$$

with arbitrary $g(x)$. This $f(x, s)$ represents a nonlinear perturbation located at $s=0$ that gives $\delta$-function kicks to the particle as it circulates around the ring. Following the smooth approximation, we first obtain

$$
\langle f\rangle_{s}=\frac{\epsilon}{2 \pi n} \sum_{k=1}^{n} g\left[\sqrt{2 J \beta} \cos \left(\phi+2 \pi \frac{k m}{n}\right)\right],
$$

where $\beta=\beta(0)$. We then decompose $\langle f\rangle_{s}$ into a detuning term plus an oscillating term according to Eq. (14). The detuning term is found using Eq. (13) to be

$$
D_{\nu}(J)=\frac{\epsilon}{4 \pi^{2}} \int_{0}^{2 \pi} d \xi g(\sqrt{2 J \beta} \cos \xi) .
$$

\section{A. Multipole magnet field error}

When the perturbation is given by a magnet multipole, we take

$$
g(x)=x^{n}, \quad \epsilon=\frac{\ell}{n !(B \rho)} \frac{\partial^{n-1} B_{y}}{\partial x^{n-1}},
$$

where $(B \rho)=P / e$ is the magnetic rigidity of the particle ( $P$ is the particle momentum, $e$ is its electric charge), and $\ell$ is the length of the multipole magnet. For a nonlinear multipole, we assume $n \geq 3$. Applying the smooth approximation, we obtain

$$
\begin{aligned}
D_{\nu}(J) & =\frac{\epsilon}{\pi}\left(\frac{J \beta}{2}\right)^{n / 2}\left\{\begin{array}{cc}
\frac{n !}{2[(n / 2) !]^{2}}, & \text { if } n=\text { even, } \\
0, & \text { if } n=\text { odd, }
\end{array}\right. \\
f_{1}(\phi, J) & =\frac{\epsilon}{\pi}\left(\frac{J \beta}{2}\right)^{n / 2} \cos n \phi,
\end{aligned}
$$

where $\beta$ is the $\beta$ function evaluated at the multipole.
In this example, we see that the detuning function $D_{\nu}(J)$ is provided by the multipole if $n$ is even. When $n$ is odd, however, the multipole contribution to the detuning vanishes, and to stabilize the beam distribution, it is necessary that an additional detuning is provided by some other source of nonlinearity, and this additional detuning must be such that $D_{\nu}(J)$ grows with $J$ at least as fast as $J^{n / 2}$ to overcome the resonance term.

Substituting Eq. (37) into Eq. (4) gives (take $n$ to be even) the equilibrium beam distribution

$$
\psi(\phi, J)=\exp \left[-\frac{J}{J_{0}}-\frac{\frac{\epsilon}{\pi J_{0}}\left(\frac{J \beta}{2}\right)^{n / 2} \cos n \phi}{\nu-\frac{m}{n}+\frac{\epsilon}{4 \pi J} \frac{n n !}{[(n / 2) !]^{2}}\left(\frac{J \beta}{2}\right)^{n / 2}}\right] .
$$

Substituting into Eq. (5) gives the quantum lifetime shortening factor

$$
\exp \left[\frac{|\epsilon| A^{n}}{\left|\pi 2^{n}\left(\nu-\frac{m}{n}\right) \frac{\sigma_{x}^{2}}{\beta}+\frac{1}{2} \frac{n n !}{[(n / 2) !]^{2}} \epsilon \sigma_{x}^{2} A^{n-2}\right|}\right] .
$$

If operated exactly on resonance, $\nu=\frac{m}{n}$, it follows that the quantum lifetime is shortened by a factor independent of the multipole strength. For an octupole magnet, for example, $n=4$, and the factor is $e^{A^{2} / 12 \sigma_{x}^{2}}$.

\section{B. Beam-beam perturbation}

Now we apply our results to the beam-beam perturbation. In a colliding-beam storage ring, the head-on collisions of the colliding beams produce a perturbation of the form (33). Consider only $x$ motion (or $y$ motion as the case may be). Assuming no dispersion at the interaction point, the beam-beam interaction can be described by [7]

$$
\epsilon=\frac{N r_{0}}{\gamma}, \quad g(x)=\int_{0}^{\infty} d t \frac{1-\exp \left(-\frac{x^{2}}{2 \sigma_{x}^{2}+t}\right)}{\sqrt{\left(2 \sigma_{x}^{2}+t\right)\left(2 \sigma_{y}^{2}+t\right)}},
$$

where $r_{0}$ is the classical radius of the particles, $\gamma$ is the relativistic Lorentz factor, and $N$ is the number of particles in a colliding-beam bunch. Again following the smooth approximation procedure, we obtain

$$
\begin{aligned}
\langle f\rangle_{s} & =\frac{\epsilon}{2 \pi n} \int_{0}^{\infty} \frac{d t}{\sqrt{\left(2 \sigma_{x}^{2}+t\right)\left(2 \sigma_{y}^{2}+t\right)}} \sum_{k=1}^{n}\left[1-e^{-2 X \cos ^{2}[\phi+2 \pi(k m / n)]}\right] \\
& =\frac{\epsilon}{2 \pi} \int_{0}^{\infty} \frac{d t}{\sqrt{\left(2 \sigma_{x}^{2}+t\right)\left(2 \sigma_{y}^{2}+t\right)}}\left[1-e^{-X} \sum_{\ell=-\infty}^{\infty}(-1)^{\ell n^{\prime}} I_{\ell n^{\prime}}(X) e^{2 i \ell n^{\prime} \phi}\right],
\end{aligned}
$$

where $X=\left(J \beta / 2 \sigma_{x}^{2}+t\right)$, and

$$
n^{\prime}= \begin{cases}n, & \text { if } n=\text { odd } \\ \frac{n}{2}, & \text { if } n=\text { even }\end{cases}
$$

Note that the results are independent of $m$. We can set $m=1$. 
Decomposing $\langle f\rangle_{s}$ into detuning and oscillating terms,

$$
D_{\nu}(J)=\frac{\epsilon}{2 \pi} \int_{0}^{\infty} \frac{d t}{\sqrt{\left(2 \sigma_{x}^{2}+t\right)\left(2 \sigma_{y}^{2}+t\right)}}\left[1-e^{-X} I_{0}(X)\right], \quad f_{1}(\phi, J)=-\frac{\epsilon}{\pi} \sum_{\ell=1}^{\infty}(-1)^{\ell n^{\prime}} \cos 2 \ell n^{\prime} \phi \int_{0}^{\infty} \frac{d t e^{-X} I_{\ell n^{\prime}}(X)}{\sqrt{\left(2 \sigma_{x}^{2}+t\right)\left(2 \sigma_{y}^{2}+t\right)}} .
$$

The beam distribution is given by substituting Eq. (43) into Eq. (4).

The extreme value of $f_{1}$ occurs at $\phi=\pi / 2$, i.e., $\hat{f}_{1}(J)=\left|f_{1}\left(\frac{\pi}{2}, J\right)\right|$. [This is because the integral over $t$ is positive; the extreme value occurs when $\cos 2 \ell n^{\prime} \phi=(-1)^{\ell n^{\prime}}$. Also, for $\hat{f}_{1}(J)$, we take the absolute value of $f_{1}\left(\frac{\pi}{2}, J\right)$ because we are interested in obtaining the maximum oscillation amplitude of $f_{1}$ and we assumed $\nu-\frac{m}{n}+D_{\nu}^{\prime}\left(\frac{A^{2}}{2 \beta}\right)>0$.] If we designate

$$
D_{\nu}=\frac{\epsilon}{2 \pi} h_{0}\left(\frac{J \beta}{2 \sigma_{x}^{2}}, \frac{\sigma_{y}}{\sigma_{x}}\right), \quad \hat{f}_{1}(J)=\frac{\epsilon}{2 \pi} h_{n}\left(\frac{J \beta}{2 \sigma_{x}^{2}}, \frac{\sigma_{y}}{\sigma_{x}}\right),
$$

then

$$
h_{0}(x, r)=\int_{0}^{1} \frac{d u}{u \sqrt{1+\left(r^{2}-1\right) u}}\left[1-e^{-x u} I_{0}(x u)\right], \quad h_{n}(x, r)=\int_{0}^{1} \frac{d u e^{-x u}}{u \sqrt{1+\left(r^{2}-1\right) u}}\left[\frac{1}{n} \sum_{k=1}^{n} e^{x u \cos [4 \pi(k / n)]}-I_{0}(x u)\right] .
$$

Note that $h_{n}(x, r)=h_{2 n}(x, r)$ if $n=$ odd. Note also that the beam-beam tune shift parameter [7] is given by

$$
\begin{aligned}
\Delta \nu_{\mathrm{BB}} & =D_{\nu}^{\prime}(0) \\
& =\left.\frac{N r_{0}}{2 \pi \gamma} \frac{\beta}{2 \sigma_{x}^{2}} \frac{\partial h_{0}(x, r)}{\partial x}\right|_{x=0}=\frac{N r_{0} \beta}{2 \pi \gamma \sigma_{x}\left(\sigma_{x}+\sigma_{y}\right)}
\end{aligned}
$$

Figure 1 shows $h_{0}(x, r)$ and $h_{n}(x, r)$ as functions of $x$ for three cases $r=\sigma_{y} / \sigma_{x}=0.1,1$, and 10, respectively. In each subfigure, the top thick curve is $h_{0}$. The three thinner curves, ordered from top to bottom, are $h_{4}, h_{3}=h_{6}$, and $h_{5}=h_{10}$, respectively. The case $r=10$ can be alternatively considered to be applied to the case $r=0.1$ but for the $y$ motion. Figure 2 shows the detuning function $\partial h_{0}(x, r) / \partial x$ as a function of $x$ for the cases (from top to bottom) $r=10,1$, and 0.1 .
Quantum lifetime is reduced by a factor

$$
\exp \left(\frac{\Delta \nu_{\mathrm{BB}}(1+4) h_{n}}{\left|\nu-\frac{m}{n}+\frac{1}{2} \Delta \nu_{\mathrm{BB}}(1+r) h_{0}^{\prime}\right|}\right)
$$

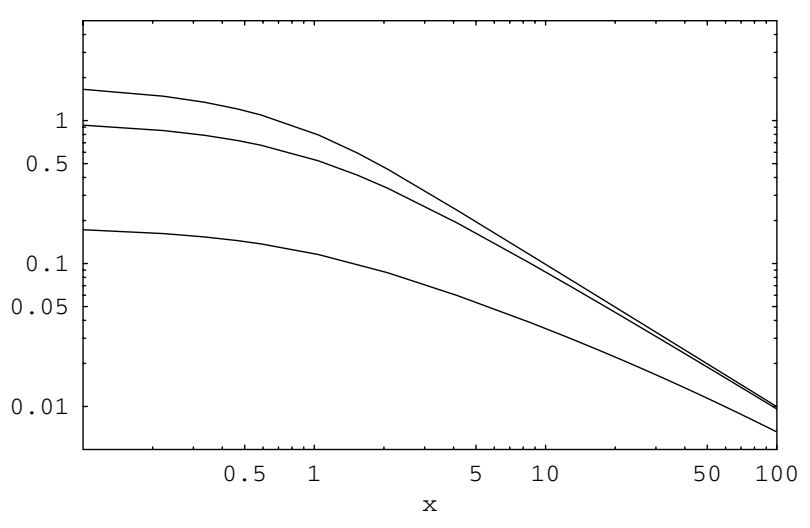

FIG. 2. Function $\partial h_{0}(x, r) / \partial x$ versus $x$.
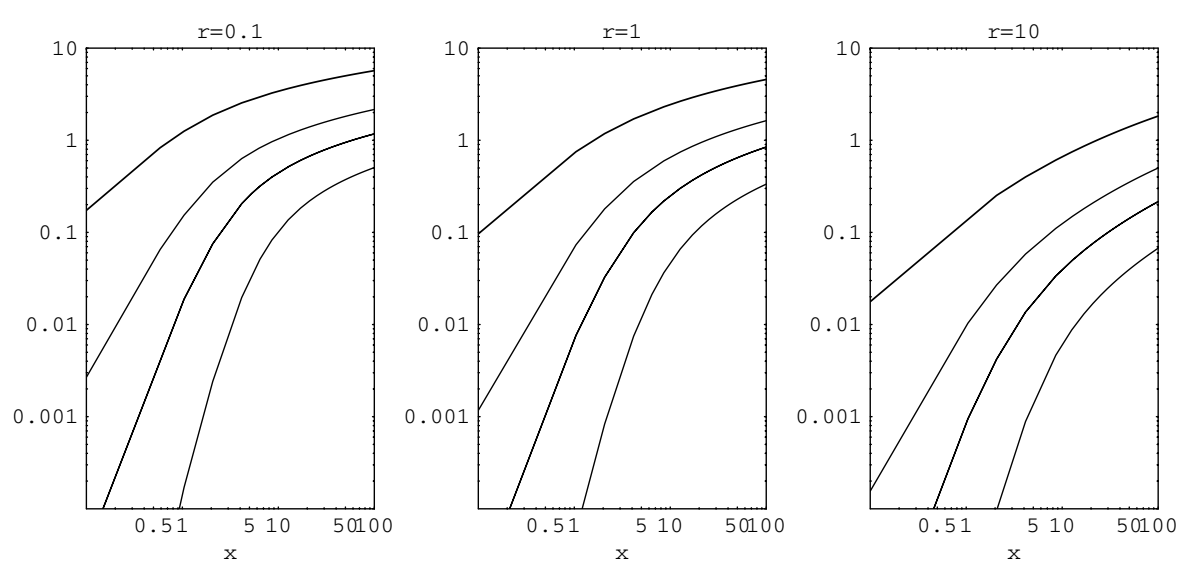

FIG. 1. Functions $h_{0}(x, r)$ and $h_{n}(x, r)$ versus $x$. 
where $\quad h_{0}^{\prime}=\left[\partial h_{0}(x, r) / \partial x\right], \quad h_{n}=h_{n}(x, r) \quad$ with $\quad x=$ $\left(A^{2} / 4 \sigma_{x}^{2}\right), r=\sigma_{y} / \sigma_{x}$. As a numerical example, taking $\Delta \nu_{\mathrm{BB}}=0.05, n=4, \nu-\frac{m}{4}=0.01, A=6 \sigma_{x}$, and $r=$ 0.1 , we have $h_{4}=0.99, h_{0}^{\prime}=0.11$ and the quantum lifetime is shortened by a factor of 70 compared with the value without the nearby resonance.

\section{ACKNOWLEDGMENTS}

This work was supported by the Department of Energy, Contract No. DE-AC03-76SF00515.

[1] A. Schoch, CERN Report No. 57-23, 1958.
[2] Matthew Sands, SLAC Report No. 121, 1970.

[3] E. D. Courant and H. S. Snyder, Ann. Phys. (Paris) 3, 1 (1958).

[4] S. Chandrasekhar, Rev. Mod. Phys. 15, 1 (1943).

[5] Distribution (4) is obtained with constraints (19) and (20). One might extend the regime of applicability by venturing the following: first invert the relation $K_{0}(J)=$ $\frac{1}{R}\left(\nu-\frac{m}{n}\right) J+\left[D_{\nu}(J) / R\right]$ to obtain $J=K_{0}^{-1}\left(K_{0}\right)$, i.e., $J$ expressed in terms of $K_{0}$, and then the equilibrium distribution might be written as $\exp \left[-K_{0}^{-1}(K) / J_{0}\right]$.

[6] A.W. Chao, Lecture Notes in Physics (Springer-Verlag, Berlin, 1986), Vol. 296, p. 51.

[7] See, for example, K. Hirata and J. M. Jowett, in Handbook of Accelerator Physics and Engineering, edited by Chao and Tigner (2002), 2nd ed., pp. 136, 146. 